suit local circumstances such as department layout, patient demand levels, skill mix and interests of GPs practitioners and the accessibility of community primary care services. Pathways to redirect patients with non-urgent primary care problems to community primary care services were also used, with local variation in protocols based on staffing, patient demand and links to community primary care services. Local clinical leads and managers need to consider which pathway(s) may best suit their local context and needs. Consistency of terminology used to describe pathways between EDs and primary care services is necessary for multi-site evaluation, quality improvement and performance measurement.

\section{THE TYPES AND EFFECTS OF FEEDBACK RECEIVED BY EMERGENCY AMBULANCE STAFF: A SYSTEMATIC MIXED STUDIES REVIEW WITH NARRATIVE SYNTHESIS}

${ }^{1}$ Caitlin Wilson, ${ }^{1}$ Jonathan Benn, ${ }^{2}$ Gillian Janes, ${ }^{3}$ Rebecca Lawton. ${ }^{1}$ University of Leeds; ${ }^{2}$ Manchester Metropolitan University; ${ }^{3}$ University of Leeds/Bradford Institute for Health Research

\subsection{6/emj-2020-rcemabstracts.34}

Aims/Objectives/Background The phenomenon of feedback is well-researched within the wider healthcare context, where it is suggested that feedback can improve patient care and patient safety by enhancing clinical performance and staff mental health. Within prehospital care, reviews have been conducted for automated feedback from defibrillators and debrief after simulation, but not on the wider concept of feedback. The aim of this systematic review is to identify, describe and synthesize the published literature on the types and effects of feedback received by emergency ambulance staff. PROSPERO CRD42020162600.

Methods/Design The search strategy consisted of three facets: ambulance staff synonyms, feedback synonyms and feedback content. Databases searched from inception were MEDLINE, EMBASE, AMED, PsycInfo, HMIC, CINAHL and Web of Science. Studies were included if they were qualitative or quantitative empirical research exploring the concept of feedback, i. e. the systematised provision of information to emergency ambulance staff regarding their performance within prehospital practice. Study quality was appraised using the Mixed Methods Appraisal Tool and data analysed using narrative synthesis. Results/Conclusions The search strategy yielded 2424 articles excluding duplicates. 94 studies met the inclusion criteria after full-text review, of which 36 fleetingly mentioned increased feedback as a solution to improving specific circumstances (e. g. decision-making, burnout). The remaining 58 studies consisted of 48 interventional prehospital feedback studies, 8 noninterventional studies and 2 feasibility studies. Narrative synthesis revealed a wide range of prehospital feedback types with the majority of studies focusing on performance or patient outcome feedback. The effects of feedback encompassed both personal and professional development, such as improved decision-making, reflection and closure. Mechanisms of providing prehospital feedback varied from electronic dashboards to case reviews and performance appraisals. Further empirical research is required to explore whether the published literature reflects current prehospital practice, as well as to determine the when, what, how and why of ambulance staff desiring feedback.

\section{THE RELATIONSHIP BETWEEN INTRACRANIAL MRI ABNORMALITIES AND POST-CONCUSSIVE SYMPTOMS IN ED PATIENTS WITH A NORMAL CT: AS DEMONSTRATED ON THE RIVERMEAD POST CONCUSSION SYMPTOM QUESTIONNAIRE (RPQ)}

${ }^{1}$ Daniel Whitehouse, ${ }^{2}$ Sophie Richter, ${ }^{2}$ Stefan Winzeck, ${ }^{2}$ Evgenios N Kornaropoulos, ${ }^{2}$ Tilak Das, ${ }^{3}$ Thijs Vande Vyvere, ${ }^{4}$ Jan Verheyden, ${ }^{5}$ Guy B Williams, ${ }^{6}$ Marta M Correia, ${ }^{2}$ David K Menon, ${ }^{1}$ Virginia FJ Newcombe, ${ }^{7}$ CENTER-TBI MRI Sub-Study Participants and Investigators. 'University of Cambridge, University Division of Anaesthesia; ${ }^{2}$ Division of Anaesthesia, Department of Medicine, University of Cambridge, Cambridge, UK; ${ }^{3} 1$ Research and Development, Icometrix, Kolonel Begaultlaan 1b/12, 3012 Leuven, Belgium 2- Department of Radiology, Antwerp University Hospital and University of Antwerp; ${ }^{4}$ Research and Development, Icometrix, Kolonel Begaultlaan 1b/12, 3012 Leuven, Belgium; ${ }^{5}$ Department of Clinical Neurosciences, Wolfson Brain Imaging Centre, University of Cambridge; ${ }^{6}$ MRC Cognition and Brain Sciences Unit, University of Cambridge; ${ }^{7}$ CENTERTBI MRI Sub-Study Participants and Investigators

\subsection{6/emj-2020-rcemabstracts.35}

Aims/Objectives/Background Mild traumatic brain injury (TBI) is common presentation to the ED. Mild, however, is a misnomer with $10-40 \%$ of patients suffering from post-concussion symptoms for months to years following injury. ${ }^{1}{ }^{2}$ Patients often re-present to primary care or ED with these symptoms, and the role of repeat imaging in this cohort remains uncertain. Aims: assess TBI patients discharged from the ED with no acute intracranial findings on CT head scan, who subsequently had a research-driven MRI and documented 3-month RPQ, to determine the association between ongoing post-concussion symptoms and MRI pathology.

Methods/Design 91 patients in the CENTER-TBI dataset met the inclusion criteria. ${ }^{3}$ Mann-Whitney $\mathrm{U}$ test used to compare 3-month RPQ and MRI findings. Numbers and percentages of patients with RPQ $>35$ and $>19$ presented owing to a score of 35 predicting moderate to severe activity limitation, ${ }^{4}$ and 19 representing mean RPQ in patients with diagnosed postconcussion syndrome (PCS). ${ }^{2}$

Results/Conclusions 15/91 CT-ve (16.5\%) patients had abnormalities on acute MRI (2 intraparenchymal haemorrhages, 13 Diffuse Axonal Injury (DAI)). No significant difference between median 3 month RPQ between MRI -ve (2.00 [IQR 0.00 - 14.00] and MRI +ve (0.00 [IQR 0.00 - 8.50]) patients $(p=0.51$, Mann-Whitney $U$ test $)$. Of patients with a RPQ $>35$, only $1 / 8(12.5 \%)$ had a +ve MRI. Of patients with a RPQ >19 2/14 (14.3\%) had +ve MRI, both DAI.

No difference was found between RPQ scores of MRI positive and negative patients, suggesting no significant relationship between ongoing symptomology following mild TBI and gross MRI findings in patients with a negative acute CT. This study is limited by a small number of patients with positive neuroimaging and a lack of quantitative MRI data. Further prospective research is required to assessing a larger patient cohort and more sensitive imaging modalities to examine the utility of repeat neuroimaging in patients with ongoing concussive symptoms.

\section{REFERENCES}

1. Polinder $S$, Cnossen $M C$, Real RGL, et al. A multidimensional approach to postconcussion symptoms in mild traumatic brain injury. Front. Neurol 2018;9:1113. doi:10.3389/fneur.2018.01113

2. Ingebrigtsen T, Waterloo K, Marup-Jensen $S$, et al. Quantification of post-concussion symptoms 3 months after minor head injury in 100 consecutive patients. J Neurol 1998;245:609-12. doi:10.1007/s004150050254

3. Maas AIR, Menon DK, Steyerberg EW, et al. Collaborative European neurotrauma effectiveness research in traumatic brain injury (CENTER-TBI): A prospective longitudinal observational study. Neurosurgery 2015;76:67-80. doi:10.1227/ NEU.0000000000000575 